Pediat. Res. 4: 375-381 (1970)

\title{
ABSTRACTS Meeting of
The Society for Pediatric Research
}

\author{
Atlantic City, New Jersey, May 1, 1970
}

Plenary Sessions

15 Antenatal Infection and Prematurity: Poverty and Race. Richard Naeye and William Blanc. Pennsylvania State Univ. Coll. of Med. and Columbia Univ. Coll. of Phys. and Surg., Depts. of Path., Hershey, PA, and New York City.

An excess number of low birth weight infants in poor families accounts for much of the excessive perinatal death rates recorded in the US. Fetal undernutrition accounts for some of this low birth weight but infection appears to play a more important role by inducing early delivery. Necropsy material was examined from 1002 consecutive autopsies on stillborn and newborn infants. Using income data and a US Government poverty index, cases were classified by economic status. A surprising $36 \%$ showed anatomic evidences of antenatal infection in the placenta, membranes, umbilical cord and/or newborn organs. In $77 \%$ of the infected cases, infant organs were involved. Although most mothers received antenatal care in the same clinic, the incidence of infection related directly to economic status and race. Infection rates in the poorest group were: blacks $55 \%$, whites $45 \%$, Puerto Ricans $45 \%$. Rates in the most prosperous clinic group were: blacks $38 \%$, whites $27 \%$, Puerto Ricans $36 \%$. Rates in semiprivate and private patients were somewhat lower.

In the majority of cases, infection appeared to antedate labor and membrane rupture. The overall rate for premature membrane rupture was only $11 \%$; individual rates were highest in blacks and lowest in whites. Newborn organ cultures were undertaken in most cases. A specific agent was identified in $55 \%$, gram-negative organisms being most common. Mean gestational ages were: infected infants 29.2 weeks; placental/membrane infection only 38.8 weeks; no infection 32.3 weeks. $20 \%$ of the cases with negative cultures had received antibiotics.

16 Growth, Inheritance and Environment. George G. Graham and Blanca Adrianzen T. The Johns Hopkins Univ., Baltimore, MD, and British American Hosp., Lima, Peru.

Current thinking attributes permanent effects on stature and head size to finite periods of severe malnutrition in early life. One study [Lancet $i: 1,1967$ ] found no difference in later size between the severely malnourished and their 'healthy' siblings. In 13 mestizo families we have followed the growth of 15 cases of third degree infant malnutrition and of 18 next-born siblings given an adequate diet in a controlled environment during the first 18-24 months of life and then returned to their habitual hostile environment. Whereas at 2 years height was well below the US 3rd percentile in all the former and between the 3rd and 90th in the latter (mean at 10 th-25th percentile), 2 to 5 years later there is no difference between the two groups or between them and other 'healthy' siblings, suggesting that the environment has wiped out the advantages of a better start. Most of the severely malnourished have recovered to between the 3rd and 10th percentiles and their siblings have dropped to the same range. Head circumference at a given height is not significantly different between the 2 groups, both having a smaller circumference than US children (50th percentile) of the same height after 6 months of age. Relatively larger deficits in head size previously attributed to malnutrition probably represent racial differences. 'Catch-up' growth in stature and head size can continue for many years and eventual size is much more the result of genetic potential and the duration of undernutrition than of any single episode of severe malnutrition.

17 In vitro Fertilization of Mouse Ova: Development of Normal Progeny. ANIL B. MukHerJeE and Maimon M. Gohen. Buffalo Children's Hosp., Dept. of Ped., State Univ. of New York at Buffalo.

In vitro fertilization of mammalian ova has been achieved in several species but the subsequent development of derived embryos to term has almost invariably failed. We have succeeded in obtaining normal progeny from the implantation of in vitro fertilized mouse ova that have been maintained in tissue culture to the blastocyst stage ( 3 days). Fertilization was accomplished as described by Whrtringham and the embryos were cultured in a modified Whitten and Biggers medium. Of 253 eggs exposed to capacitated sperm, 67 were fertilized as evidenced by the 2-cell stage, and 25 developed to blastocysts. 23 blastocysts were implanted into surrogate mothers and 11 apparently normal fertile offspring were obtained. The implantations were designed so that the coat color of the progeny derived from in vitro or in vivo fertilizations could be unequivocally identified. Birth usually occurred 17-18 days following the implantation of the blastocysts, which, including 3 days of in vitro growth, yielded a normal gestation time of 20-21 days. This technique may provide a model in vitro system for the study of many aspects of development, including growth requirements, the possible effects of teratogenic and mutagenic agents, and X-chromosome inactivation through the early cleavage stages in various mammals. 
18 Intragenic Complementation in Hybrid Cells Derived from Human Diploid Fibroblasts: A Model System for the Study of Genetic Heterogeneity. HenRy L.

Nadler, Glaramma M. Ghacko and Martin RaChMELER. Northwestern Univ. Med. Sch., Children's Mem. Hosp., Chicago, IL.

The technique of cell hybridization has been utilized to study genetic heterogeneity of the galactose-1phosphate uridyl transferase (transferase) locus. Fibroblasts cultivated from skin biopsies of seven unrelated galactosemics had no demonstrable transferase activity. Hybrids were made from all possible combinations of these cell lines. Cells were mixed in equal numbers in the presence of UV inactivated Sendai virus, incubated for $2 \mathrm{~h}$ at $37^{\circ} \mathrm{C}$ and cultured. Hybrid formation could be detected cytologically within $24 \mathrm{~h}$. Transferase active cells were selected by periodic changes of glucose and galactose concentrations in the medium. In several of the pairs the percentage of hybrids was notably increased by this procedure. Transferase activity was detected in three instances and in each case one mutant line was involved.

These data provide the first example of intragenic complementation in fused human hybrid cells. In line with observations of complementation in other systems, these data indicate that the transferase enzyme is composed of subunits. This system provides a model by which genetic heterogeneity can be investigated at the cellular level. Those familial metabolic disorders that are detectable in tissue culture are amenable to study by this approach.

19 Identification of Type Independent Nephritogenic Streptococcal Antigens in Acute Glomerulonephritis. ANTONia Ty, Inge SAGEL and KurT LANGe. Renal Serv. and Lab., Depts. of Med. and Ped., New York Med. Coll., New York, NY. (introduced by Miriam Lending)

Fluorescein labeled immunoglobin (IgG) fractions of sera from patients with acute poststreptococcal glomerulonephritis (AGN) stained the glomerular basement membrane of renal tissue obtained during the early phase of the disease. The amount of fluorescence present on the glomeruli of patients with AGN when stained with their own labeled IgG or the IgG of other patients with AGN was determined by quantitative fluorometry. Using the spectrofluorometric methods it was quantitatively shown that the staining capacity of these IgG fractions was reduced after they had been preabsorbed with disrupted nephritogenic group A streptococci (type 12, 49, and a non-typeable strain) or their isolated plasma membranes. In contrast disrupted streptococci or the plasma membranes of types considered frequently nephritogenic (e.g. 12) when obtained from patients without AGN did not reduce the staining capacity of nephritic sera nor did other types $(3,4,6,14$ and 25$)$ obtained from patients without nephritis. These streptococci or their plasma membrane markedly depresssing straining even on biopsies coming from patients with AGN due to an infection with streptococci of different serologic type. By sucrose density gradient ultracentrifugation of the nephritogenic plasma membranes (type 12) an antigenic phosphate buffer saline soluble component of 120,000 molecular weight was obtained. These findings suggest that a specific nephritogenic antigen is contained in the plasma membrane of certain streptococci and that this antigen is not necessarily connected with serologic types. Our results further indicate that a common antigen is present in the plasma membranes of different serotypes.

20 Characterization of 'Natural' Infection and Immunization-induced Anti-capsular Antibodies to H. influenza Type b. J.B. Robbins, R. SahneERson, L.P.RoDRIGUes and J.C.PARke, Jr. Dept. of Ped., Albert Einstein Coll. of Med., New York City, and Charlotte Memorial Hosp., Charlotte, NG. Anti type b capsular antibodies were examined in the sera of normal newborns, infants and adults by hemagglutination. No antibody activity was detected in $2 \%$ of cord sera, $45 \%$ of infant sera from $2-30$ months and $5 \%$ of adult individuals. Of 20 infants and children with severe $H$. influenza type b disease, 17 had no detectable antibody in 'acute' sera. All individuals demonstrated a rise in antibodies during convalescence.

Nineteen adult individuals were immunized with $50 \mu \mathrm{g}$ of the purified capsular substance. No febrile response, leukocytosis, or adventitious urinary findings were detected in the following $24 \mathrm{~h}$. All individuals responded with anti-capsular antibodies in 7-14 days with a maximum level reached in 21 days and maintained for at least 90 days.

Complement-dependent bactericidal and mouseprotection assays were done on several sera from the 'acute' and 'convalescent' samples of infected infants and from the pre- and post-injection samples of the immunized adults. Sera of both groups showed a rise in bactericidal activity which could be blocked by preincubation with the purified capsular substance. Postimmune and convalescent sera showed a low but detectable mouse-protection activity. By these criteria, it may be concluded that a similar antibody response is induced by immunization with the purified capsular substance as occurs after infectious disease with $H$. influenza type b.

21 White Villus Disease: A Form of Hypo-beta Lipoproteinemia in Infancy. Jorn C. PARTIN and WILliam K. Sahubert. The Children's Hosp. Res. Found., Cincinnati, Ohio.

Biochemical, immunological and light and electron microscopical (EM) observations of a 16-month-old girl with a form of hypo-beta lipoproteinemia are presented. She developed nearly fatal diarrhea and marasmus during the 1st month of life; after resuscitation by protein hydrolysate-glucose infusions, steatorrhea persisted (25 g stool fat/day), and growth retardation developed. Anemia, acanthocytosis and neurological signs were absent. At 16 months of age, biopsy specimens of jejunal (3) and ileal (1) tissue were snowy white: villus tips were slightly expanded. Light microscopy demonstrated massive triglyceride (TG) storage in absorptive epithelium but not in generative epithelium or lamina propria. EM proved the TG was stored in epithelial vesicles originating from endoplasmic reticulum; TG was reduced but not eliminated by 4-day glucose supplemented fasts. Serum lipoprotein electrophoresis in agarose demonstrated reduction in low density lipoprotein (LDL) and absent very low density lipoprotein (VLDL). Immunoelectrophoresis (reagent: goat anti-human beta lipoprotein, Weizmann Inst.) revealed reduction in beta lipoprotein; double diffusion precipitin analysis showed the reagent to be against 4 lipid staining serum components. Numbered from the antigen well, components 1 and 2 were reduced and 4 was undetectable. These findings are in contrast to a second case of hypo-beta lipo- 
proteinemia in whom component 1 is absent and 3 and 4 reduced, who stores TG not in the epithelium but in the villus core. The results suggest the existence of multiple functional beta lipoproteins deficiencies each of which produce distinctive histological abnormalities.

Fasting Hypoglycemia and Metabolic Acidosis Associated with a Deficiency of Hepatic Fructose-1,6-diphosphatase (FDPase) Activity-A Nerw Syndrome. Lester Baker and Albert I. Winegrad. Children's Hosp. of Philadelphia, PA.

At age 5 months this female infant presented with fasting hypoglycemia, hepatomegaly, metabolic acidosis, and hyperuricemia. Glucagon produced no rise in blood glucose after fasting, but a normal response in the fed state. Liver glycogen was $1.4 \%$, and normal hepatic levels of glucose-6-phosphatase, phosphorylase, a-glucosidase, amylo-1,6-glucosidase and glycogen synthetase activities were found (Dr. BARBARA I. GROWN). Severe fatty infiltration of the liver was observed, similar in appearance to that found in her brother who died suddenly elsewhere at age 6 months.

The patient was restudied at age $41 / 2$ years because of repeated episodes of fasting hypoglycemia and metabolic acidosis. Oral glucose, galactose, and leucine tolerance tests produced normal responses in blood glucose and plasma insulin levels. Despite the lack of any history suggestive of hereditary fructose intolerance, oral fructose produced hypoglycemia without hyperinsulinemia, hypophosphatemia, and hyperuricemia. Oral glycerol reproduced the effects of oral fructose. The findings suggested the possibility of an enzymatic defect in gluconeogenesis at the level of FDPase. Normal levels of hepatic fructose-1-phosphate and fructose-1,6-diphosphate aldolase activity were observed; however, no FDPase activity could be demonstrated. No evidence of an enzyme inhibitor was found. The patient was shown to have impaired urinary hydrogen ion excretion following an ammonium chloride load. This syndrome may represent the consequences of an inherited defect in FDPase activity.

Clinical and Experimental Relationships Between Cholestasis and Abnormal Hepatic Lymphatics. Oystein Aagenaes, Bart Cuderman, Helge SigSTAD, Arnold S. Leonard, William Krivit and HARVEY L. Sharp. Univ. of Oslo, Norway, and Univ. of Minn. Sch. of Med., Depts. of Ped. and Surg., Minneapolis, Minn.

A new syndrome with severe infantile cholestasis and lymphedema of the lower extremities was found in 9 Norwegian sibships (20 patients) and 1 American (2 siblings) of Norwegian origin. Lymph vessel hypoplasia was present in the extremities. Hepatic lymphangiography with subcapsular injection of $\mathrm{Au}^{198}$ during a non-cholestatic phase in one adult patient with the inherited disorder demonstrated no isotope clearance to the thoracic duct. Two experimental models were devised to study the relationship between bile flow, bile acid secretion and the hepatic lymphatics. I. The common bile duct was obstructed in cats and the hepatic lymph was collected. Bile acids increased from less than $2 \mu \mathrm{g} / \mathrm{ml}$ to a maximum $200-250 \mu \mathrm{g} / \mathrm{ml}$ within $2 \mathrm{~h}$. Both bile acids and bilirubin drain via the lymph prior to any rise in the serum. II. The hepatic lymphatics were obstructed at all local lymph nodes in the portal region and liver bile was collected during the same day or the next. Control animals were sham operated. Bile flow increased slightly the same day and to more than $50 \%$ the following day along with an increase in bile salt secretion in the lymph obstructed cat. No difference was found in the protein excretion or the osmolarity between experimental and control cats. Therefore, the physiology of the hepatic lymph flow is intimately related to bile secretion in our experimental models. Congenital lymph abnormalities in the liver may cause cholestasis.

$24 A$ New Experimental Model of Myocarditis. G.R. Noren, E.F.Jankus, N.A.Staley and J.E. Stevenson. Univ. of Minn. Sch. of Med., Coll. of Vet. Med. and Chas. T. Miller Hosp., Minneapolis and St.Paul, Minn. (introduced by R.V.Lucas)

Round heart disease in the turkey is a spectrum of left ventricular dilatation and hypertrophy associated with endocardial fibroelastosis. Most losses from this disease occur in the second week of life with a low rate of mortality, secondary to congestive heart failure, continuing until market age. This experiment of nature was studied in two affected flocks of 1074 and 135 birds, from age 1 day to 12 months.

\begin{tabular}{lcc}
\hline & $\begin{array}{c}\text { Round heart } \\
\text { flocks }\end{array}$ & $\begin{array}{c}\text { Control } \\
\text { flock }\end{array}$ \\
\hline $\begin{array}{l}\text { Gross abnormalities/ } \\
\text { no. studied }\end{array}$ & $307 / 1209$ & $1 / 1600$ \\
$\begin{array}{l}\text { Microscopic abnormalities/ } \\
\text { no. studied }\end{array}$ & $64 / 97$ & $1 / 60$ \\
\hline
\end{tabular}

During the first week of life the turkey appears clinically well. However, in the second and third week the more severely affected bird appears wasted and may succumb to the illness. Grossly the heart is flaccid and there is biventricular dilatation. Microscopically, there is pancarditis. Electron microscopy in 26 birds revealed fragmented mitochondria and myofilaments, in addition to virus-like particles $90-150 \mu$ in size.

In turkeys that survive this acute phase, histologic evidence of inflammation gradually subsides with increasing amounts of collagen and elastic fiber deposition in the endocardium. In the most severely affected maturing turkey, there is mitral insufficiency, congestive failure and a gross and microscopic picture identical to 'primary' endocardial fibroelastosis.

25 Congenital Anomalies Associated with Parental LSD Ingestion. Cheston M.BerLIN and Gecil B. Jacobson. Ghildren's Hosp. of DC and George Washington Univ. Sch. of Med. (introduced by F. Heald).

Since 1968 a prospective study (124 pregnancies) has been underway evaluating the reproductive effects of lysergic acid diethylamide (LSD). Pregnant women are admitted to the study after parental use of LSD has been ascertained. A detailed social and medical history is obtained. Serial cultures for chromosomes are done on peripheral blood, skin, and amnion at the time of delivery. The infant, or, if abortion occurs, the conceptus, is examined at the time of delivery or abortion. Infants are followed serially with pediatric evaluation and cultures for chromosome analysis of peripheral blood and skin. Careful growth and developmental data, urinalysis, and complete blood counts are obtained. Of the 124 pregnancies, there were 65 abortions: 3 missed, 7 spontaneous, 1 incomplete, and 54 
therapeutic (36 D and C, 18 hypertonic saline). There were 59 live births. Of the 14 abortuses suitable for examination, 4 showed severe congenital defects with midline fusion defects of the central nervous system. Of the 59 live births, there are 5 infants with severe congenital anomalies: 3 infants with myelomeningocele and hydrocephalus, 1 with hydrocephalus only, 1 with congenital heart disease and developmental and growth retardation. A sixth infant had congenital amputation of both feet, multiple cutaneous hemangiomas, and intrauterine growth retardation. Although this is a high risk maternity population with multiple drug use, frequent infectious disease, communal living, and marginal nutrition, the possibility of LSD as a mutagen must be seriously considered.

26 Virus-like Particles in Kidney Biopsies of Children with Systemic Lupus. JosepH R. Goodman, JaNET Beck and Garolyn F. Piel. Dept. of Ped., Univ. of California, San Francisco, and V.A. Hosp.

Ultramicroscopic demonstration of virus-like particles within glomerular endothelial cytoplasm occurs frequently enough in adults to be considered of diagnostic significance. Forty percutaneous renal biopsies done in the last 12 years in 24 children with onset of lupus from ages $3 \frac{1}{2}$ to 16 years have been retrospectively reviewed for prevalence of such particles. In 9 patients with active lupus nephritis, virus-like particles were seen in only 2 of 16 biopsies, questionably in 3 . In 8 patients with minimal nephritis, 8 of 16 biopsies were positive and 3 questionable. Of 3 children with membranous lesions, 2 biopsies were positive in 1, 1 was questionable; 4 biopsies normal by light microscopy were positive. This retrospective study found no significant correlation between presence of particles with presence of renal disease or electron dense masses. In 2 patients, no particles were seen in the first biopsy and appeared 5 years later in 1 , and questionably in 6 years in the other. In 1 patient, no particles were seen initially but became positive 5 years later; postmortem 5 months later revealed periarteritis nodosa. Forty biopsies in patients with other renal disease were negative. (Supported in part by USPHS Grant RR 00079.)

Recurrent Respiratory Illness in Survivors of the Idiopathic Respiratory Distress Syndrome. EugENE W. Outerbridge, Bernadette Nogrady, Pierre H. Beaudry and Leo Stern. McGill Univ.Montreal Children's Hosp. Res. Inst.

It is commonly believed that infants who survive the idiopathic Respiratory distress syndrome (RDS) go on to complete resolution of the pulmonary disease and do not show any permanent after effects. This report concerns 6 infants with severe recurrent respiratory disease, in the first year of life, following complete recovery from RDS. All 6 had clinical, radiological, and arterial blood gas findings of RDS in the neonatal period. In 4 cases the disease was of sufficient severity $\left(\mathrm{PaO}_{2}<50 \mathrm{~mm} \mathrm{Hg}\right.$ in $\left.100 \% \mathrm{O}_{2}\right)$ to require mechanical assistance with a negative pressure respirator. All 6 showed complete clinical and biochemical recovery, as well as radiologic clearing of the disease prior to discharge from hospital. Subsequently all have had repeated episodes of a bronchiolitic type illness with radiologic evidence of pulmonary overdistention and air trapping. This has resulted in a total of 22 hospital admissions between 5 weeks and 21 months of age. Three of the 6 required ventilatory assistance with a respirator for periods of 10-23 days during these admissions. Sweat chlorides and serum immunoglobulins were normal in 4 infants tested. With one exception, there is no family history of allergy. Tuberculous skin tests have been negative in all. Follow-up radiologic examinations show varying degrees of increased markings, peri-bronchial thickening and pulmonary overdistention as evidence of persistent pulmonary disease. The incidence of later respiratory disease in RDS survivors is not known. This experience suggests a need to re-evaluate our present concepts regarding the longterm pulmonary future of RDS survivors.

$28 \quad$ Neurological and Psychometric Evaluation of Rh Erythroblastotic Infants Surviving Intrauterine Blood Transfusions. W. OH, J.ARBIT, E.R.BLONSKY and S. Gassell. Michael Reese Hosp. and Northwestern Univ. Med. Sch., Chicago, IL, and Harbor Gen. Hosp., UCLA'Sch. of Med., Torrance, GA.

During a $4 \frac{1}{2}$-year period, 107 intrauterine blood transfusions were performed in 59 severely sensitized $\mathrm{Rh}$ negative mothers. Twenty-nine infants were delivered alive at 33-36 weeks of gestation with a mean birth weight of $2420 \mathrm{~g}$. Seventeen of these infants survived. Two infants required resuscitation at birth, $4 \mathrm{had}$ respiratory distress syndrome, 2 had neonatal hypoglycemia treated promptly with $10 \%$ glucose in water intravenously, 2 had hypocalcemia, and 2 had inspissated bile syndrome. All infants required 1 or more exchange transfusion during the first week of life. None had serum indirect bilirubin in excess of $20 \mathrm{mg} \%$. At 12-54 months of age, 11 infants were recalled for neurological psychometric and electroencephalographic examinations. Each infant was matched with a control as to sex, race, birth weight, gestation and home environment.

Neurological and electroencephalographic findings were within normal range. The I.Q. or D.Q. of the Rh survivors using Stanford-Binet, Peabody or Catell tests, was $94.9 \pm 2.9$ (SEM), while those of controls were $98.9 \pm 2.4$. The data indicates that in severe $\mathrm{Rh}$ erythroblastotic infants, treated with intrauterine blood transfusion along with intensive neonatal care, normal neurological and developmental performance may be achieved.

29 Staphylococcal Scalded Skin Syndrome (SSS): Experimental Model and Isolation of a New Exfoliative Toxin (ET). Marian E. Melush, Lowell A. Glasgow and Michael D.Turner. Depts. of Ped., Micro. and Med., Univ. of Rochester Sch. of Med., Rochester, NY.

Infection with phage group II staphylococci may be associated with an expanded SSS including generalized exfoliation (Ritter's syndrome), bullous impetigo and scarlatiniform rash. An experimental model of SSS has been developed. Phage group II staph isolated from 18 patients representing each of these clinical entities produced exfoliation in newborn mice which clinically and histologically resembled the human disease; 23 strains of other phage groups did not. The experimental model was utilized to evaluate therapy: Methicillin prevented exfoliation if given early; pharmacological doses of hydrocortisone resulted in exfoliation with only $1 / 100$ to $1 / 1000$ the inoculum which was effective in non-treated animals.

A sterile filtrate of phage group II staph reproduced the exfoliation in newborn mice. The staphylococcal 
product in the filtrate producing exfoliation was termed ET and has been partially characterized. Initial studies show that ET: (1) is soluble in $50 \%$ sat. $\mathrm{NH}_{4}$ $\mathrm{SO}_{4}$ - and precipitates at $80 \%$ sat.; (2) passes a ' 50,000 MW' membrane but is retained by a ' 10,000 MW' membrane; (3) migrates with staph ahemolysin $(\alpha \mathrm{H})$ on Pevikon block electrophoresis; (4) has the same elution volume as $\alpha \mathrm{H}$ on Sephadex G100; (5) is a macromolecular ampholyte with an isoelectric point of approximately 6.0 and has a molecular weight of the same order as $\alpha H ;(6)$ sediments with $\alpha \mathrm{H}$ in sucrose but is separable from $\alpha \mathrm{H}$ by isoelectric focusing; and, (7) is not neutralized by specific $\alpha \mathrm{H}$ antibody.

These data demonstrate that the skin changes associated with phage group II staph infection are mediated by a newly recognized toxin. Treatment should be directed against the toxin-producing staph. Corticosteroids may be detrimental.

30 Lung Function Following Correction of Spinal Curves. Daniel C. Shannon, EDWARd P. Riseborough, Laercio M.Valenca and Homayoun Kazemi. Harvard Med. Sch., Massachusetts Gen. Hosp., Children's Service, and Pulmonary Unit, Boston, Mass. (introduced by Joseph B.Warshaw).

Lung function was studied in 10 patients 13-24 years old with spinal curves $\left(52-120^{\circ}\right)$ before and 9-12 months after correction $\left(18-60^{\circ}\right)$. Vital capacity (VC) and total lung capacity (TLC) were measured by helium dilution technique. Predicted values were based on height. Gas exchange was determined at rest, exercise and at $\mathrm{FiO}_{2}=1.0$. Alveolar samples were collected from the expiratory side of a Hans Rudolph valve and arterial samples were drawn from a Riley needle and analyzed for $\mathrm{P}_{\mathrm{O}_{2}}$ and $\mathrm{P}_{\mathrm{CO}_{2}}$. Minute ventilation $\dot{\mathrm{V}}_{\mathrm{E}}$ ) was measured for $3 \mathrm{~min}$; physiologic dead space was determined $\left(\mathrm{VD}=\mathrm{VT} \times \mathrm{PaCO}_{2}-\mathrm{P}_{\mathrm{ECO}_{2}} / \mathrm{PaCO}_{2}\right)$. With curves $<65^{\circ}, V^{\circ}$ and TLG were normal initially; with curves $>65^{\circ}$ VG and TLC were impaired with larger curves $(r=0.92)$. After correction VG and TLC did not change. $\dot{\mathrm{V}}_{E}$ at rest fell from 8.5 (SEM 1.2) to 6.3 (SEM 0.91) $\mathrm{L} / \mathrm{min}$ BTPS and $\mathrm{PaCO}_{2}$ of 35 and $34 \mathrm{~mm}$ $\mathrm{Hg}$ respectively (SEM 1). VD fell from $155 \mathrm{ml}$ (SEM 22) to $90 \mathrm{ml}$ (SEM 15). PaO, at rest averaged $87 \mathrm{~mm}$ $\mathrm{Hg}$ (SEM 2) initially and fell to $77 \mathrm{mg} \mathrm{Hg}$ (SEM 3) with exercise. After correction $\mathrm{PaO}_{2}$ rose to $93 \mathrm{~mm} \mathrm{Hg}$ (SEM 3) at rest and fell to $85 \mathrm{~mm} \mathrm{Hg}$ with exercise. A-a $\Delta \mathrm{O}_{2}$ at rest improved from 27 to $21 \mathrm{~mm} \mathrm{Hg}$. A-a $\Delta \mathrm{O}_{2}$ at $\mathrm{FiO}_{2} 1.0$ remained at $75 \mathrm{~mm} \mathrm{Hg}$ following correction. Abnormal gas exchange with spinal curves was due to ventilation perfusion inequality. Following correction, although lung volumes do not change, distribution of function within those volumes was improved, manifest by a $42 \%$ fall in VD and relief of hypoxemia.

31 Adaptation of the Developing Rat Brain to Nutritional Statuts. ARnold L. Smith, Children's Hosp. Med. Center, Boston, Mass.; H.S.Sarterthwarte and L.Sokoloff. N.I.M.H., Bethesda, MD. (introduced by David H. Smith).

Glucose is generally thought to be the main oxidative substrate for the brain. Owen et al. have shown, however, that during starvation of adults the brain utilizes $\mathrm{D}(-)-\beta$-hydroxybutyrate $(\mathrm{BOH})$ to a significant degree, while decreasing its utilization of glucose. Using microfluorometric techniques we have found that the ketonemia and hypoglycemia characteristic of starvation are also present in the postnatal rat. Previous work from this laboratory has shown that the infant rat brain can oxidize BOH during this period. This oxidation is catalyzed by $\mathrm{D}(-)-\beta$-hydroxybutyrate dehydrogenase (HBDH), a phospholipid dependent, NAD + requiring mitochondrial enzyme whose activity is measured by quantitative methods developed in this laboratory. Postnatally the blood $\mathrm{BOH}$ concentration increases 20 -fold with nursing, but falls rapidly with weaning. Brain $\mathrm{HBDH}$ activity parallels both the rise and fall in the blood BOH level. Artificial feeding of a high carbohydrate diet instead of the usual high fat diet (rat milk) effects a 30\% decrease in the blood $\mathrm{BOH}$ concentration and a $50 \%$ decrease in brain $\mathrm{HBDH}$ activity. Fasting of infants at 20 and 23 days when the $\mathrm{BOH}$ and $\mathrm{HBDH}$ levels are normally falling produces a $50 \%$ decrease in blood glucose and a $100 \%$ increase in blood $\mathrm{BOH}$ and $\mathrm{HBDH}$ activity.

Thus it appears that during development the blood glucose is low and the brain adapts its oxidative metabolism to an available alternative substrate ( $\mathrm{BOH})$. This enzymatic adaptation to the oxidation of $\mathrm{BOH}$ appears functionally significant in that it is not accompanied by gross cerebral malfunction.

32 Cystinosis: Biochemical, Morphological, and Clinical Studies. Joseph D. Schulman, Vemon G.Wong, Kathryn H. Bradley, William H. Olson and J.Edwin Seegmiller. NIH and Univ. of California, San Diego, CA. (introduced by William L. Nyhan).

Cystine crystals in conjunctival macrophages were visualized by electron microscopy in organelles which accumulate exogenously supplied ferritin, indicating continuing endocytosis by the crystal-containing lysosomes. Leukocytes and cultured fibroblasts from cystinosis carriers show compartmentalization of cystine within a subcellular fraction sedimentable at 270,000 $\mathrm{g} / \mathrm{min}$, demonstrating that cystine compartmentalization cannot be secondary to crystal formation in cystinosis. Normal levels of cystine were found in cystinotic brain and lymphocytes, which contain lysosomes but are not actively endocytic. Pulsing experiments with cystine-- ${ }^{35} \mathrm{~S}$ show that the cystine pool in cystinotic fibroblasts has a half life of about $6 \mathrm{~h}$, and that the cystine concentration gradient across the lysosomal membrane is very steep. Other amino acids are not stored in abnormal amounts in lysosomes of cystinotic leukocytes as demonstrated by analysis of their free amino acid content, although an unidentified ninhydrin-reactive peak was noted which was absent in controls; these observations fail to support the hypothesis of an abnormal shared transport system for cystine and certain other amino acids in the cystinotic lysosomal membrane. The permeability of this membrane to Damino acids and peptides with molal volumes near that of cystine $(140 \mathrm{ml})$ is being investigated in cultured fibroblasts by the method of CoHN [J. exp. Med. 129: 201]; so far differences between cystinotic and normal cells have not been noted. Perpetual blast lines from cystinotics have been developed (free $1 / 2$ cystine $4-6$ $\mu \mathrm{g} / \mathrm{g}$ protein) which will permit for the first time large scale harvesting of cystinotic lysosomes for study. Labeling of amniotic fluid cells with cystine ${ }^{35} \mathrm{~S}$ has enabled us to successfully diagnose the carrier state for nephropathic cystinosis in utero.

33 Effect of Antenatal Drug Administration on Aggregation of Platelets of Newborn Infants. DonaLD G. Gorby, Ghris Ty L.Zirbel, Mark S. Gibson 
and Irving Schulman. Dept. of Ped., The Abraham Lincoln Sch. of Med., Univ. of Illinois, Chicago, IL.

Platelet aggregation was studied in platelet-rich plasma (PRP) prepared from samples of maternal and cord blood taken immediately after delivery. When mothers had received no drugs for analgesia, adenosine diphosphate- and collagen-induced aggregation curves of platelets from the infants and from the mothers were virtually identical and were within the normal range. When mothers (18) had received drugs (acetylsalicylic acid, promethazine, alphaprodine and meperidine) prior to delivery, 17 of 18 of the cord blood samples showed a marked decrease in collagen-induced platelet aggregation. By contrast, in paired PRP samples from these mothers abnormalities of collagen-induced platelet aggregation were found in only $25 \%$, and these changes were minimal. These findings suggested that the infants' platelets were more susceptible to the druginduced suppression of collagen aggregation than were platelets of the mothers. Dose response curves to promethazine added in vitro demonstrated a markedly increased susceptibility of newborn platelets when compared with those of the mothers. These results indicate that a variety of drugs given to mothers before delivery may alter platelet aggregation of the newborn infants, resulting in significant impairment of the plateletmediated phase of hemostasis.

Plasma 17-OH Progesterone in Maternal and Umbilical Cord Plasma in Children, and in Congenital Adrenal Hyperplasia (CAH): Application to Neonatal Diagnosis of CAH. MoRris R. JenNer, MeLVIN M. Grumbach and Selna L. Kaplan. Univ. of California, San Francisco Med. Center, San Francisco, CA.
The concentration of plasma $17 \alpha-\mathrm{OH}$ progesterone (17-OHP) was determined utilizing a ligand binding radioassay at delivery in maternal (8) and umbilical venous (8) blood and in the blood of newborn infants (8), children (12), adult males (13), and pre- and posttreatment in 6 patients with CAH. Adult male values (mean $=0.106 \mu \mathrm{g} / 100 \mathrm{ml}$ ) are in agreement with the data of STROTr et al. [J.clin. Invest. 48: 930, 1969]. Mean concentration in maternal plasma at delivery was $0.365 \mu \mathrm{g} / 100 \mathrm{ml}$ and in cord plasma $1.64 \mu \mathrm{g} / 100 \mathrm{ml}$. Twelve newborns (age 1-7 days) demonstrated a rapid fall in concentration of plasma 17-OHP within the first day of life (range $<0.100-0.125 \mu \mathrm{g} / 100 \mathrm{ml}$ ). Twelve normal children (age 5-11 years) had a mean level of $0.036 \mu \mathrm{g} / 100 \mathrm{ml}$ (range 0.016-0.059). In contrast, 5 children (age 4 days -7 years) and an adult (age 26 years) with untreated $\mathrm{CAH}$ had a mean value of $12.8 \mu \mathrm{g} / 100 \mathrm{ml}$ (range 2.4-33.0 $\mu \mathrm{g} / 100 \mathrm{ml}$ ). A 4-dayold female pseudohermaphrodite, the youngest subject with CAH, had a plasma $17 . \mathrm{OHP}$ of $5.7 \mu \mathrm{g} / 100 \mathrm{ml}$. In 1 infant with CAH the concentration of plasma 17-OHP fell from $11.4 \mu \mathrm{g} \%$ to $0.12 \mu \mathrm{g} \%$ on cortisone therapy, and in a female, age $2-7 / 12$ years, from 33.0 $\mu \mathrm{g} \%$ to $0.148 \mu \mathrm{g} / 100 \mathrm{ml}$. In 4 patients with $\mathrm{CAH}$, changes in plasma testosterone paralleled those in 17$\mathrm{OHP}$. The results suggest that estimation of plasma $17-\mathrm{OHP}$ in a $0.2 \mathrm{ml}$ sample by this method permits rapid identification of the 21 hydroxylase deficiency form of CAH by the 2nd day of life and is useful in assessing the adequacy of glucocorticoid treatment. The high concentration of plasma 17-OHP in cord blood is attributable to the capacity of the placenta to convert maternal and fetal steroid precursors to 17OHP.

INDEX OF ABSTRACTS

(Numbers following entries refer to abstract number)

Aagenaes, O. 23

adenoids 1

Adrianzen, T.B. 16

amniocentesis 25

amniotic fluid 4

antibody, rubella 7

Arbit, J. 28

BAKER, L. 22

BEAUDRY, P.H. 27

BEGK, J. 26

BerLin, C. M. 25

bile 23

Blanc, W. 15

BLONSKY, E.R. 28

blood 33

BRADLEY, K.H. 32

brain 31

Caldwell, B. M. 13

carbohydrate metabolism 11, 22

care, pediatric 2

CASSELl, S. 28

cell hybridization 18 central nervous system 28,31

Chabor, A. 10

Ghacko, C. M. 18

Ghapman, E. M. 14

child care 10,13

children 26

cholestasis 23

chromosomes 25

Cochran, W. 9

Cohen, M.M. 17

community health program 10

Condon, A. 3

congenital adrenal hyperplasia 34

congenital anomalies 25

congenital rubella 7

COOPER, L.Z. 7

Corby, D. G. 33

CRAWFORD, J.D. 14

Cuderman, B. 23

cystine 32

cystinosis 32

deafness 7

deoxyadenosyl- $\mathrm{B}_{12} 12$ development 31

developmental pharmacology 33

diagnosis, prenatal 4

DiMauro, S. 3

Dorfman, A. 4

Embryo, mouse 17 endocardial fibroelastosis 24 enzyme 22

erythroblastosis 9

erythrocytes 6

exfoliation 29

Fertilization, in vitro 17

fetal transfusions 9

fetus 3

fibrinolysis 5

fibrin split products 5

Florman, A. L. 7

fructose-1,6-diphosphatase 22

Galactose 1-phosphate uridyl

transferase 18

genetic disease 12,21 